\title{
A sufficient and necessary condition for the convergence of the sequence of
} successive approximations to a unique fixed point II

Tomonari Suzuki ${ }^{1,2^{*}}$ and Badriah Alamri²

"Correspondence:

suzuki-t@mns.kyutech.ac.jp

'Department of Basic Sciences,

Faculty of Engineering, Kyushu Institute of Technology, Tobata Kitakyushu, 804-8550, Japan

2Department of Mathematics, Faculty of Science, King Abdulaziz

University, Jeddah, Saudi Arabia

\begin{abstract}
Using the concept of a Boyd-Wong contraction, we obtain a simple, sufficient, and necessary condition for the convergence of the sequence of successive approximations to a unique fixed point.
\end{abstract}

MSC: $54 \mathrm{H} 25$

Keywords: Banach contraction principle; Boyd-Wong contraction; contraction; fixed point; successive approximation

\section{Introduction}

The Banach contraction principle is a very forceful tool in nonlinear analysis.

Theorem 1 (Banach [1] and Caccioppoli [2]) Let $(X, d)$ be a complete metric space and let $T$ be a contraction on $X$, that is, there exists $r \in[0,1)$ such that $d(T x, T y) \leq r d(x, y)$ for all $x, y \in X$. Then the following holds:

(A) $T$ has a unique fixed point $z$ and $\left\{T^{n} x\right\}$ converges to $z$ for any $x \in X$.

In $[3,4]$ we studied (A) and obtained the following. See also $[5,6]$.

Theorem $2([3,4])$ Let $T$ be a mapping on a complete metric space $(X, d)$. Then (A), (B), and $(\mathrm{C})$ are equivalent:

(B) $T$ is a strong Leader mapping, that is, the following hold:

- For $x, y \in X$ and $\varepsilon>0$, there exist $\delta>0$ and $v \in \mathbb{N}$ such that

$$
d\left(T^{i} x, T^{j} y\right)<\varepsilon+\delta \quad \Longrightarrow \quad d\left(T^{i+v} x, T^{j+\nu} y\right)<\varepsilon
$$

for all $i, j \in \mathbb{N} \cup\{0\}$, where $T^{0}$ is the identity mapping on $X$.

- For $x, y \in X$, there exist $v \in \mathbb{N}$ and a sequence $\left\{\alpha_{n}\right\}$ in $(0, \infty)$ such that

$$
d\left(T^{i} x, T^{j} y\right)<\alpha_{n} \quad \Longrightarrow \quad d\left(T^{i+v} x, T^{j+v} y\right)<1 / n
$$

for all $i, j \in \mathbb{N} \cup\{0\}$ and $n \in \mathbb{N}$.

\section{Springer}


(C) There exist a $\tau$-distance $p$ and $r \in(0,1)$ such that $p\left(T x, T^{2} x\right) \leq r p(x, T x)$ and $p(T x, T y)<p(x, y)$ for all $x, y \in X$ with $x \neq y$.

We cannot tell that Theorem 2 is simple. Motivated by this fact, in this paper, we obtain a simpler condition equivalent to (A).

In 1969, Boyd and Wong proved a very interesting fixed point theorem. See [7]. The concept of a Boyd-Wong contraction plays an important role in this paper. Indeed, using this concept, we give a condition equivalent to (A); see Theorem 9 below. We will find that Theorem 3 is an essential generalization of Theorem 1 in some sense; see Theorem 11 and Example 12 below.

Theorem 3 (Boyd and Wong [8]) Let $(X, d)$ be a complete metric space and let $T$ be a BoydWong contraction on $X$, that is, there exists a function $\varphi$ from $[0, \infty)$ into itself satisfying the following:

(i) $\varphi$ is upper semicontinuous.

(ii) $\varphi(t)<t$ for every $t \in(0, \infty)$.

(iii) $d(T x, T y) \leq \psi(d(x, y))$ for all $x, y \in X$.

Then (A) holds.

Later, in 1975, Matkowski proved the following generalization of Theorem 1. Interestingly, while Theorem 3 and Theorem 4 look similar, we will find that Theorem 4 is similar to Theorem 1, not Theorem 3, in some sense; see Theorem 13 below.

Theorem 4 (Matkowski [9]) Let $(X, d)$ be a complete metric space and let $T$ be a Matkowski contraction on $X$, that is, there exists a function $\psi$ from $[0, \infty)$ into itself satisfying the following:

(i) $\psi$ is nondecreasing.

(ii) $\lim _{n} \psi^{n}(t)=0$ for every $t \in(0, \infty)$.

(iii) $d(T x, T y) \leq \psi(d(x, y))$ for all $x, y \in X$.

Then (A) holds.

We introduce two more interesting theorems. Theorem 5 is a generalization of Theorem 3 and Theorem 6 is a generalization of Theorems 4 and 5 .

Theorem 5 (Meir and Keeler [10]) Let $(X, d)$ be a complete metric space and let $T$ be a Meir-Keeler contraction on $X$, that is, for every $\varepsilon>0$, there exists $\delta>0$ such that

$$
d(x, y)<\varepsilon+\delta \quad \Longrightarrow \quad d(T x, T y)<\varepsilon
$$

for all $x, y \in X$. Then (A) holds.

Theorem 6 (Ćirić [11], Jachymski [12] and Matkowski [13, 14]) Let $(X, d)$ be a complete metric space and let $T$ be a CJM contraction on $X$, that is, the following hold:

(i) For every $\varepsilon>0$, there exists $\delta>0$ such that $d(x, y)<\varepsilon+\delta$ implies $d(T x, T y) \leq \varepsilon$.

(ii) $x \neq y$ implies $d(T x, T y)<d(x, y)$.

Then (A) holds. 


\section{Preliminaries}

Throughout this paper, we denote by $\mathbb{N}, \mathbb{Z}$, and $\mathbb{R}$ the sets of positive integers, integers, and real numbers, respectively. For $t \in \mathbb{R}$, we denote by $[t]$ the maximum integer not exceeding $t$. For an arbitrary set $A$, we denote by $\sharp A$ the cardinal number of $A$.

Let $(X, d)$ be a metric space. We denote by $\operatorname{Cont}(X, d), \operatorname{BWC}(X, d), \operatorname{MC}(X, d), \operatorname{MKC}(X, d)$, and $\operatorname{CJMC}(X, d)$ the sets of all contractions, all Boyd-Wong contractions, all Matkowski contractions, all Meir-Keeler contractions, and all CJM contractions on $(X, d)$, respectively. We know

$$
\operatorname{Cont}(X, d) \subset \operatorname{BWC}(X, d) \subset \operatorname{MKC}(X, d) \subset \operatorname{CJMC}(X, d)
$$

and

$$
\operatorname{Cont}(X, d) \subset \operatorname{MC}(X, d) \subset \operatorname{CJMC}(X, d) .
$$

In the proof of our main result, we use the following.

Lemma 7 Let $X$ be a set, let $z$ be an element of $X$ and let $f$ be a function from $X \backslash\{z\}$ into $(0, \infty)$. Define a function $\rho$ from $X \times X$ into $[0, \infty)$ by

$$
\rho(x, y)= \begin{cases}0 & \text { if } x=y, \\ f(x) & \text { if } x \neq y, y=z, \\ f(y) & \text { if } x \neq y, x=z, \\ \max \{f(x), f(y)\} & \text { if } x \neq y, x \neq z, y \neq z .\end{cases}
$$

Then $(X, \rho)$ is a complete metric space.

Proof It is obvious that $\rho(x, y)=0 \Leftrightarrow x=y$, and $\rho(x, y)=\rho(y, x)$. We also note that

$$
\rho(x, y)=\max \{\rho(x, z), \rho(y, z)\} \quad \text { for all } x, y \in X \text { with } x \neq y \text {. }
$$

Let $x, y, w$ be three distinct elements of $X \backslash\{z\}$. We have

$$
\begin{aligned}
\rho(x, z) & \leq \max \{\rho(x, z), \rho(y, z)\}=\rho(x, y) \leq \rho(x, y)+\rho(z, y), \\
\rho(x, y) & =\max \{\rho(x, z), \rho(y, z)\} \leq \rho(x, z)+\rho(y, z), \\
\rho(x, y) & =\max \{\rho(x, z), \rho(y, z)\} \\
& \leq \max \{\max \{\rho(x, z), \rho(w, z)\}, \max \{\rho(y, z), \rho(w, z)\}\} \\
& =\max \{\rho(x, w), \rho(y, w)\} \leq \rho(x, w)+\rho(y, w) .
\end{aligned}
$$

So $\rho$ satisfies the triangle inequality. Therefore $(X, \rho)$ is a metric space. Finally, in order to show the completeness of $(X, \rho)$, let $\left\{x_{n}\right\}$ be a Cauchy sequence in $X$. In the case where $\sharp\left\{n: x_{n}=y\right\}=\infty$ for some $y \in X,\left\{x_{n}\right\}$ obviously converges to $y$. In the case where $\sharp\left\{n: x_{n}=\right.$ $y\}<\infty$ for any $y \in X$, we can choose a subsequence $\left\{x_{g(n)}\right\}$ of $\left\{x_{n}\right\}$ such that $x_{g(n)}$ are all 
different. Then we have

$$
0=\lim _{\substack{m \neq n \\ m, n \rightarrow \infty}} \rho\left(x_{g(m)}, x_{g(n)}\right)=\lim _{\substack{m \neq n \\ m, n \rightarrow \infty}} \max \left\{\rho\left(x_{g(m)}, z\right), \rho\left(x_{g(n)}, z\right)\right\}
$$

Thus, $\left\{x_{g(n)}\right\}$ converges to $z$. Hence $\left\{x_{n}\right\}$ converges to $z$. Therefore $(X, \rho)$ is complete.

We denote by $\Psi$ the set of all functions $\psi$ satisfying (i) and (ii) of Theorem 4 .

Lemma 8 Let $\varphi$ and $\psi$ belong to $\Psi$. Then a function $\eta$ from $[0, \infty)$ defined by $\eta(t)=$ $\max \{\varphi(t), \psi(t)\}$ also belongs to $\Psi$.

Proof It is obvious that $\eta$ is nondecreasing. Since $\varphi(t)<t$ and $\psi(t)<t$ for $t \in(0, \infty)$, we have $\eta(t)<t$ for $t \in(0, \infty)$. Fix $t \in(0, \infty)$. Define a mapping $v$ from $\mathbb{N}$ into $\mathbb{N} \cup\{0\}$ by

$$
v(n)=\sharp\left\{k \in \mathbb{Z}: 0 \leq k<n, \psi\left(\eta^{k}(t)\right)=\eta^{k+1}(t)\right\},
$$

where $\eta^{0}(t)=t$. Without loss of generality, we may assume $\lim _{n} v(n)=\infty$. Since $\eta^{n}(t) \leq$ $\psi^{\nu(n)}(t)$, we obtain $\lim _{n} \eta^{n}(t)=0$. Therefore $\eta \in \Psi$.

\section{Main results}

We prove our main results.

Theorem 9 Let $(X, d)$ be a complete metric space and let $T$ be a mapping on $X$. Then (A) and (D) are equivalent.

(D) There exists a complete metric $\rho$ on $X$ such that $\rho \geq d$ and $T \in \operatorname{BWC}(X, \rho)$.

Proof $(\mathrm{D}) \Rightarrow(\mathrm{A})$ : We assume (D). Then Theorem 3 shows that there exists a unique fixed point $z$ of $T$ and that for every $x \in X,\left\{T^{n} x\right\}$ converges to $z$ in $(X, \rho)$. Since the topology of $(X, \rho)$ is stronger than that of $(X, d),\left\{T^{n} x\right\}$ converges to $z$ in $(X, d)$. Hence (A) holds.

(A) $\Rightarrow$ (D): We assume (A). Define functions $\alpha$ from $X \backslash\{z\}$ into $(0, \infty), \ell$ from $X \backslash\{z\}$ into $\mathbb{Z}, k$ from $X \backslash\{z\}$ into $\mathbb{N} \cup\{0\}$ and $f$ from $X \backslash\{z\}$ into $(0, \infty)$ by

$$
\begin{aligned}
& \alpha(x)=\max \left\{d\left(T^{n} x, z\right): n \in \mathbb{N} \cup\{0\}\right\}, \\
& \ell(x)=\left[\log _{4} \alpha(x)\right], \\
& k(x)=\max \left\{n \in \mathbb{N} \cup\{0\}: T^{n} x \neq z, \ell\left(T^{n} x\right)=\ell(x)\right\}, \\
& f(x)=2^{2 \ell(x)+4}-2^{2 \ell(x)+3-k(x)} .
\end{aligned}
$$

We note

$$
d(x, z) \leq \alpha(x), \quad \alpha(T x) \leq \alpha(x), \quad \lim _{n \rightarrow \infty} \alpha\left(T^{n} x\right)=0
$$

and

$$
\ell(T x) \leq \ell(x), \quad \lim _{n \rightarrow \infty} \ell\left(T^{n} x\right)=-\infty
$$


for $x \in X \backslash\{z\}$. Define a metric $\rho$ on $X$ by (1). Then by Lemma 7, $(X, \rho)$ is a complete metric space. We shall show $\rho \geq d$. In the case where $x \neq z$, we have

$$
\begin{aligned}
\rho(x, z) & =2^{2 \ell(x)+4}-2^{2 \ell(x)+3-k(x)} \geq 2^{2 \ell(x)+4}-2^{2 \ell(x)+3}=2 \times 4^{\ell(x)+1} \\
& >2 \times 4^{\log _{4} \alpha(x)}=2 \alpha(x) \geq 2 d(x, z)>d(x, z) .
\end{aligned}
$$

In the case where $x \neq y, x \neq z$, and $y \neq z$, we have

$$
\begin{aligned}
\rho(x, y) & =\max \{\rho(x, z), \rho(y, z)\}>\max \{2 d(x, z), 2 d(y, z)\} \\
& \geq d(x, z)+d(y, z) \geq d(x, y) .
\end{aligned}
$$

In both cases, we obtain $\rho \geq d$.

Next, we shall show that $T \in \operatorname{BWC}(X, \rho)$. Define a function $\varphi$ from $[0, \infty)$ into itself by

$$
\varphi(t)= \begin{cases}0 & \text { if } t=0, \\ 0 & \text { if } 2^{2 \ell+2} \leq t<2^{2 \ell+3} \text { for some } \ell \in \mathbb{Z}, \\ 2^{2 \ell+2} & \text { if } 2^{2 \ell+4}-2^{2 \ell+3} \leq t<2^{2 \ell+4}-2^{2 \ell+2} \\ & \text { for some } \ell \in \mathbb{Z}, \\ 2^{2 \ell+4}-2^{2 \ell+4-k} & \text { if } 2^{2 \ell+4}-2^{2 \ell+3-k} \leq t<2^{2 \ell+4}-2^{2 \ell+2-k} \\ & \text { for some } \ell \in \mathbb{Z}, k \in \mathbb{N} .\end{cases}
$$

We note that $\varphi$ is well defined because

$$
\begin{aligned}
{[0, \infty) } & =\{0\} \sqcup(0, \infty)=\{0\} \sqcup \bigsqcup_{\ell \in \mathbb{Z}}\left[2^{2 \ell+2}, 2^{2 \ell+4}\right) \\
& =\{0\} \sqcup \bigsqcup_{\ell \in \mathbb{Z}}\left(\left[2^{2 \ell+2}, 2^{2 \ell+3}\right) \sqcup\left[2^{2 \ell+3}, 2^{2 \ell+4}\right)\right) \\
& =\{0\} \sqcup \bigsqcup_{\ell \in \mathbb{Z}}\left(\left[2^{2 \ell+2}, 2^{2 \ell+3}\right) \sqcup \bigsqcup_{k \in \mathbb{N} \cup\{0\}}\left[2^{2 \ell+4}-2^{2 \ell+3-k}, 2^{2 \ell+4}-2^{2 \ell+2-k}\right)\right),
\end{aligned}
$$

where ' $\sqcup$ ' represents 'disjoint union'. It is obvious that $\varphi(t)<t$ for $t \in(0, \infty)$ and $\varphi$ is right continuous. We note that $\varphi$ is strictly increasing on the range of $\rho$ because the range of $\rho$ is a subset of

$$
\{0\} \cup\left\{2^{2 \ell-4}-2^{2 \ell-3-k}: \ell \in \mathbb{Z}, k \in \mathbb{N} \cup\{0\}\right\}
$$

and

$$
2^{2 \ell-4}-2^{2 \ell-3-k}<2^{2 \ell^{\prime}-4}-2^{2 \ell^{\prime}-3-k^{\prime}}
$$

for $\ell, \ell^{\prime} \in \mathbb{Z}$ and $k, k^{\prime} \in \mathbb{N} \cup\{0, \infty\}$ with $\ell<\ell^{\prime} \vee\left(\ell=\ell^{\prime} \wedge k<k^{\prime}\right)$, where $2^{-\infty}=0$, ' $\vee$ ' represents 'logical or' and ' $\wedge$ ' represents 'logical and'. Let $x$ and $y$ be two distinct elements of $X \backslash\{z\}$. We consider the following three cases:

- $T x=z$ 
- $T x \neq z$ and $k(x)=0$;

- $T x \neq z$ and $k(x) \in \mathbb{N}$.

In the first case, we have

$$
\rho(T x, z)=0 \leq \varphi(\rho(x, z)) .
$$

In the second case, noting $\ell(T x)<\ell(x)$, we have

$$
\begin{aligned}
\rho(T x, z) & =2^{2 \ell(T x)+4}-2^{2 \ell(T x)+3-k(T x)}<2^{2 \ell(T x)+4} \leq 2^{2 \ell(x)+2} \\
& =\varphi\left(2^{2 \ell(x)+4}-2^{2 \ell(x)+3}\right)=\varphi(\rho(x, z)) .
\end{aligned}
$$

In the third case, noting $\ell(T x)=\ell(x)$ and $k(T x)=k(x)-1$, we have

$$
\begin{aligned}
\rho(T x, z) & =2^{2 \ell(T x)+4}-2^{2 \ell(T x)+3-k(T x)}=2^{2 \ell(x)+4}-2^{2 \ell(x)+4-k(x)} \\
& =\varphi\left(2^{2 \ell(x)+4}-2^{2 \ell(x)+3-k(x)}\right)=\varphi(\rho(x, z)) .
\end{aligned}
$$

We have shown $\rho(T x, T z)=\rho(T x, z) \leq \varphi(\rho(x, z))$ for all $x \in X \backslash\{z\}$. Using this and the strict monotony of $\varphi$ on the range of $\rho$, we obtain

$$
\begin{aligned}
\rho(T x, T y) & \leq \max \{\rho(T x, z), \rho(T y, z)\} \leq \max \{\varphi(\rho(x, z)), \varphi(\rho(y, z))\} \\
& =\varphi(\max \{\rho(x, z), \rho(y, z)\})=\varphi(\rho(x, y)) .
\end{aligned}
$$

Therefore $T \in \operatorname{BWC}(X, \rho)$.

From Theorem 9, we obtain the following.

Corollary 10 Let $(X, d)$ be a complete metric space and let $T$ be a mapping on $X$. Then (A), (E), (F), and (G) are equivalent.

(E) There exists a complete metric $\rho$ on $X$ such that the topology of $(X, \rho)$ is stronger than that of $(X, d)$ and $T \in \operatorname{BWC}(X, \rho)$.

(F) There exists a complete metric $\rho$ on $X$ such that the topology of $(X, \rho)$ is stronger than that of $(X, d)$ and $T \in \operatorname{MKC}(X, \rho)$.

(G) There exists a complete metric $\rho$ on $X$ such that the topology of $(X, \rho)$ is stronger than that of $(X, d)$ and $T \in \operatorname{CJMC}(X, \rho)$.

Proof It is obvious that $(E) \Rightarrow(F) \Rightarrow(G)$. The proof of $(G) \Rightarrow(A)$ is almost the same as that of $(D) \Rightarrow(A)$. Since $(E)$ is weaker than $(D)$, we have $(A) \Rightarrow(E)$ by Theorem 9 .

We note that $(E)$ is much simpler than $(B)$ and $(C)$.

\section{Additional results}

In the previous section, we have showed that (D) is equivalent to (A). In this section, we will show that the condition $(\mathrm{H})$ on contractions is not equivalent to $(\mathrm{A})$.

Theorem 11 Let $(X, d)$ be a complete metric space and let $T$ be a mapping on $X$. Then $(\mathrm{H}) \Rightarrow(\mathrm{A})$ holds . 
$(\mathrm{H})$ There exists a complete metric $\rho$ on $X$ such that the topology of $(X, \rho)$ is stronger than that of $(X, d)$ and $T \in \operatorname{Cont}(X, \rho)$.

Proof The proof of $(\mathrm{D}) \Rightarrow(\mathrm{A})$ works.

The following example tells that $(\mathrm{A}) \Rightarrow(\mathrm{H})$ does not hold.

Example 12 ([4]) Let $A$ be the set of all real sequences $\left\{a_{n}\right\}$ such that $a_{n} \in(0, \infty)$ for $n \in \mathbb{N}$, $\left\{a_{n}\right\}$ is strictly decreasing, and $\left\{a_{n}\right\}$ converges to 0 . Let $H$ be a Hilbert space consisting of all the functions $x$ from $A$ into $\mathbb{R}$ satisfying $\sum_{a \in A}|x(a)|^{2}<\infty$ with inner product $\langle x, y\rangle=$ $\sum_{a \in A} x(a) y(a)$ for all $x, y \in H$. Put $d(x, y)=\langle x-y, x-y\rangle^{1 / 2}$. Define a complete subset $X$ of $H$ by

$$
X=\{0\} \cup\left(\bigcup_{a \in A}\left\{a_{n} e_{a}: n \in \mathbb{N}\right\}\right),
$$

where $e_{a} \in H$ is defined by $e_{a}(a)=1$ and $e_{a}(b)=0$ for $b \in A \backslash\{a\}$. Define a mapping $T$ on $X$ by

$$
T 0=0 \quad \text { and } \quad T\left(a_{n} e_{a}\right)=a_{n+1} e_{a} .
$$

Then (A) holds. However, $(\mathrm{H})$ does not hold.

Proof It is obvious that (A) holds. Arguing by contradiction, we assume $(\mathrm{H})$. That is, there exist a metric $\rho$ on $X$ and $r \in[0,1)$ such that the topology of $(X, \rho)$ is stronger than that of $(X, d),(X, \rho)$ is complete and $\rho(T x, T y) \leq r \rho(x, y)$ for all $x, y \in X$. Since the topology of $(X, \rho)$ is stronger than that of $(X, d)$,

$$
\inf \{\rho(0, y): d(0, y)>t\}>0
$$

for every $t>0$. So, there exists a strictly increasing sequence $\left\{\kappa_{n}\right\}$ in $\mathbb{N}$ such that

$$
r^{\kappa_{n}}<\inf \{\rho(0, y): d(0, y)>1 / n\} .
$$

Then

$$
\rho(0, x) \leq r^{\kappa_{n}} \quad \Longrightarrow \quad d(0, x) \leq 1 / n
$$

holds. We choose $\alpha \in A$ such that $\alpha_{2 \kappa_{n}+1}>1 / n$. Fix $v \in \mathbb{N}$ with $r^{\kappa_{v}} \rho\left(0, \alpha_{1} e_{\alpha}\right) \leq 1$. Then we have

$$
\rho\left(0, \alpha_{2 \kappa_{v}+1} e_{\alpha}\right)=\rho\left(T^{2 \kappa_{v}} 0, T^{2 \kappa_{v}}\left(\alpha_{1} e_{\alpha}\right)\right) \leq r^{2 \kappa_{v}} \rho\left(0, \alpha_{1} e_{\alpha}\right) \leq r^{\kappa_{v}}
$$

and hence

$$
1 / \nu<\alpha_{2 \kappa_{v}+1}=d\left(0, \alpha_{2 \kappa_{v}+1} e_{\alpha}\right) \leq 1 / \nu \text {. }
$$

This is a contradiction. 
The Matkowski contraction version of $(\mathrm{H})$ is equivalent to $(\mathrm{H})$ itself.

Theorem 13 Let $(X, d)$ be a complete metric space and let $T$ be a mapping on $X$. Then $(\mathrm{H}) \Leftrightarrow$ (I) holds.

(I) There exists a complete metric $\rho$ on $X$ such that the topology of $(X, \rho)$ is stronger than that of $(X, d)$ and $T \in \operatorname{MC}(X, \rho)$.

Proof $(\mathrm{H}) \Rightarrow(\mathrm{I})$ : Obvious.

$(\mathrm{I}) \Rightarrow(\mathrm{H})$ : Assume (I). Then there exists a function $\psi$ satisfying (i)-(iii) of Theorem 4 with replacing $d:=\rho$. Define a function $\varphi$ from $[0, \infty)$ into itself by

$$
\varphi(t)=\max \{\psi(t), t / 2,-[-t]-1\} .
$$

Then from Lemma 8, $\varphi$ satisfies (i)-(iii) of Theorem 4 with replacing $d:=\rho$ and $\varphi:=\psi$. We note $0<\varphi(t)$ for $t \in(0, \infty)$. Also we note $k \leq \varphi(t)$ if $t$ satisfies $k<t \leq k+1$ for some $k \in \mathbb{N}$. We define a sequence $\left\{t_{n}\right\}_{n \in \mathbb{Z}}$. Put $t_{0}=1$ and $t_{n}=\varphi^{n}(1)$ for $n \in \mathbb{N}$. For $k \in \mathbb{N}$, we can choose $v(k) \in \mathbb{N}$ satisfying $\varphi^{\nu(k)}(k+1)=k$. So for $n \in \mathbb{N}$, there exists $\kappa(n) \in \mathbb{N}$ such that $\sum_{j=1}^{\kappa(n)-1} v(j)<n \leq \sum_{j=1}^{\kappa(n)} v(j)$, where $\sum_{j=1}^{0} v(j)=0$. We put $t_{-n}=\varphi^{\sum_{j=1}^{\kappa(n)} v(j)-n}(\kappa(n)+1)$. Then the following are obvious:

- $t_{n+1}=\varphi\left(t_{n}\right)$ for every $n \in \mathbb{Z}$,

- $\left\{t_{n}\right\}_{n \in \mathbb{Z}}$ is a strictly decreasing sequence,

- $\left\{t_{n}\right\}_{n \in \mathbb{Z}}$ converges to 0 as $n$ tends to $\infty$,

- $\left\{t_{n}\right\}_{n \in \mathbb{Z}}$ converges to $\infty$ as $n$ tends to $-\infty$.

Let $z \in X$ be a unique fixed point of $T$ and fix $r \in(0,1)$. Define a function $f$ from $X \backslash\{z\}$ into $(0, \infty)$ by

$$
f(x)=r^{n} \quad \text { if } t_{n+1}<\rho(z, x) \leq t_{n} \text { for some } n \in \mathbb{Z}
$$

Then we have

$$
f(T x) \leq r f(x) \quad \text { provided } T x \neq z
$$

Indeed $t_{n+1}<\rho(z, x) \leq t_{n}$ implies $f(x)=r^{n}$ and

$$
\rho(z, T x) \leq \psi(\rho(z, x)) \leq \varphi(\rho(z, x)) \leq \varphi\left(t_{n}\right)=t_{n+1}
$$

and hence $f(T x) \leq r^{n+1}=r f(x)$. We denote by $q$ a complete metric defined by (1) with replacing $q:=\rho$. Let $\left\{x_{n}\right\}$ be a sequence in $X$ such that $x_{n}$ are all different and $\left\{x_{n}\right\}$ converges to some $x \in X$ in $(X, q)$. From the definition of $q, x=z$ holds. Without loss of generality, we may assume $x_{n} \neq z$. Since $\lim _{n} f\left(x_{n}\right)=\lim _{n} q\left(z, x_{n}\right)=0$, we have $\lim _{n} \rho\left(z, x_{n}\right)=0$. Thus, $\left\{x_{n}\right\}$ converges to $z$ in $(X, \rho)$. Therefore the topology of $(X, q)$ is stronger than that of $(X, \rho)$, which is stronger than that of $(X, d)$. Let $x$ and $y$ be two distinct elements of $X \backslash\{z\}$. In the case where $T x=z$, we have

$$
q(T x, T z)=q(T x, z)=0 \leq r q(x, z) .
$$


In the other case, where $T x \neq z$, we have

$$
q(T x, T z)=q(T x, z)=f(T x) \leq r f(x)=q(x, z) .
$$

We obtain

$$
\begin{aligned}
q(T x, T y) & \leq \max \{q(T x, z), q(T y, z)\} \leq \max \{r q(x, z), r q(y, z)\} \\
& =r \max \{q(x, z), q(y, z)\}=r q(x, y) .
\end{aligned}
$$

Therefore $T \in \operatorname{Cont}(X, q)$.

The following result due to Bessaga [15] (see also [16]) shows that the topological condition appearing in condition $(\mathrm{H})$ cannot be removed, because otherwise the convergence of iterates in the metric space $(X, d)$ cannot be ensured.

Theorem 14 (Bessaga [15]) Let $X$ be a set and let $T$ be a mapping on $X$. Then (J) and (K) are equivalent.

(J) There exists a complete metric $\rho$ on $X$ such that $T \in \operatorname{Cont}(X, \rho)$.

(K) There exists a unique fixed point $z$ of $T$ and the set of periodic points of $T$ is $\{z\}$.

If $X$ is a metric space, then (J) is strictly weaker than (A) because (K) is strictly weaker than $(\mathrm{A})$.

In conclusion, we obtain

$$
(\mathrm{H}) \Leftrightarrow(\mathrm{I}) \quad \Longrightarrow \quad(\mathrm{A}) \Leftrightarrow(\mathrm{E}) \Leftrightarrow(\mathrm{F}) \Leftrightarrow(\mathrm{G}) \quad \Longrightarrow \quad(\mathrm{J})
$$

under the assumption that $(X, d)$ is a complete metric space. We can tell that, from this point of view, the difference between contractions and Matkowski contractions is small and the difference between contractions and Boyd-Wong contractions is very large. Therefore Matkowski contractions and Boyd-Wong contractions are essentially different. Considering the appearance of the statements, we might have considered that the difference between Boyd-Wong contractions and Matkowski contractions was small and the difference between Boyd-Wong and Meir-Keeler contractions was large.

Competing interests

The authors declare that they have no competing interests.

Authors' contributions

All authors contributed equally to the writing of this paper. All authors read and approved the final manuscript.

\section{Acknowledgements}

This project was funded by the Deanship of Scientific Research (DSR), King Abdulaziz University, under grant No. (35-130-35-HiCi). The authors, therefore, acknowledge technical and financial support of KAU. The first author is supported in part by Grant-in-Aid for Scientific Research from Japan Society for the Promotion of Science.

Received: 20 January 2015 Accepted: 24 March 2015 Published online: 24 April 2015

\section{References}

1. Banach, S: Sur les opérations dans les ensembles abstraits et leur application aux équations intégrales. Fundam Math. 3, 133-181 (1922)

2. Caccioppoli, R: Un teorema generale sull'esistenza di elementi uniti in una transformazione funzionale. Rend. Accad. Naz. Lincei 11, 794-799 (1930) 
3. Suzuki, T: A sufficient and necessary condition for the convergence of the sequence of successive approximations to a unique fixed point. Proc. Am. Math. Soc. 136, 4089-4093 (2008). MR2425751

4. Suzuki, T: Convergence of the sequence of successive approximations to a fixed point. Fixed Point Theory Appl. 2010 Article ID 716971 (2010). MR2595834

5. Leader, S: Equivalent Cauchy sequences and contractive fixed points in metric spaces. Stud. Math. 76, 63-67 (1983). MR0728197

6. Suzuki, T: Generalized distance and existence theorems in complete metric spaces. J. Math. Anal. Appl. 253, 440-458 (2001). MR1808147

7. Jachymski, J: Equivalence of some contractivity properties over metrical structures. Proc. Am. Math. Soc. 125, 2327-2335 (1997). MR1389524

8. Boyd, DW, Wong, JSW: On nonlinear contractions. Proc. Am. Math. Soc. 20, 458-464 (1969). MR0239559

9. Matkowski, J: Integrable solutions of functional equations. Diss. Math. 127, 1-68 (1975). MR0412650

10. Meir, A, Keeler, E: A theorem on contraction mappings. J. Math. Anal. Appl. 28, 326-329 (1969). MR0250291

11. Ćirić, LB: A new fixed-point theorem for contractive mappings. Publ. Inst. Math. (Belgr.) 30, 25-27 (1981). MR0672538

12. Jachymski, J: Equivalent conditions and the Meir-Keeler type theorems. J. Math. Anal. Appl. 194, 293-303 (1995). MR1353081

13. Kuczma, M, Choczewski, B, Ger, R: Iterative Functional Equations. Encyclopedia of Mathematics and Its Applications, vol. 32. Cambridge University Press, Cambridge (1990). MR1067720

14. Matkowski, J: Fixed point theorems for contractive mappings in metric spaces. Čas. Pěst. Mat. 105, 341-344 (1980). MR0597909

15. Bessaga, C: On the converse of the Banach 'fixed-point principle'. Colloq. Math. 7, 41-43 (1959). MR0111015

16. Jachymski, J: A short proof of the converse to the contraction principle and some related results. Topol. Methods Nonlinear Anal. 15, 179-186 (2000). MR1786260

\section{Submit your manuscript to a SpringerOpen ${ }^{\circ}$ journal and benefit from:}

- Convenient online submission

- Rigorous peer review

- Immediate publication on acceptance

- Open access: articles freely available online

- High visibility within the field

- Retaining the copyright to your article 